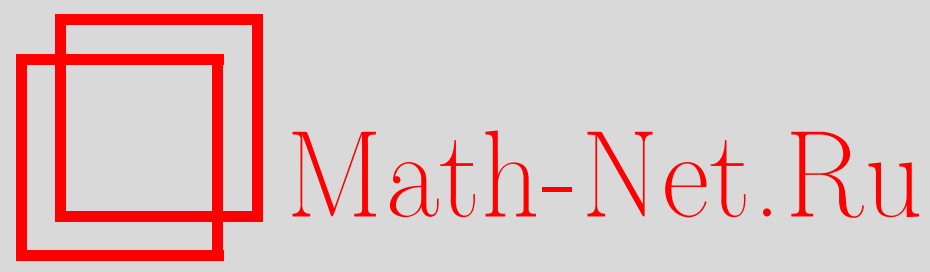

В. А. Копытцев, В. Г. Михайлов, Предельные теоремы пуассоновского типа для обобщенного линейного включения, Дискрет. матем., 2012, том 24, выпуск 3, 108-121

DOI: https://doi.org/10.4213/dm1202

Использование Общероссийского математического портала Math-Net.Ru подразумевает, что вы прочитали и согласны с пользовательским соглашением http://www . mathnet.ru/rus/agreement

Параметры загрузки:

IP : 54.198 .55 .26

26 апреля 2023 г., 18:36:28 


\title{
Предельные теоремы пуассоновского типа для обобщенного линейного включения
}

\author{
() 2012 г. В. А. Копытцев, В. Г. Михайлов
}

\begin{abstract}
Пусть $A-$ случайная матрица размера $T \times n$ над конечным полем $G F(q), D-$ некоторое множество ненулевых элементов $n$-мерного пространства $V^{n}$ над $G F(q)$, и для каждого $x \in D$ задано множество $B(x)$ элементов $T$-мерного пространства $V^{T}$ над тем же полем. Выведены достаточные условия сходимости при $n, T \rightarrow \infty$ распределения числа решений системы включений $x \in D, A x \in B(x)$ к распределениям пуассоновского типа. Полученные результаты позволили исследовать асимптотические свойства числа решений системы вида $x \in D, A x+S(x) \in B$, где $S(x)$ - произвольное отображение $V^{n}$ в $V^{T}$, и, тем самым, свойства распределения числа решений случайных полиномиальных уравнений.
\end{abstract}

\section{1. Введение}

Линейным включением (над полем $K$ ) размерности $T$ относительно $n$-мерного вектора $x$ называется запись $A x \in B$, где $A$ - матрица из элементов поля $K$ размера $T$ на $n$, a $B$ - некоторое множество $T$-мерных векторов над этим полем. Решением линейного включения является любой вектор $x$ из $n$-мерного линейного пространства $V^{n}$ над полем $K$, для которого вектор $A x$ принадлежит множеству $B$. Понятие линейного включения впервые было использовано в $[8,9]$ при изучении свойств некоторых случайных систем нелинейных уравнений над конечным полем.

Условия выполнения теорем пуассоновского типа для числа решений линейного включения $A x \in B$, удовлетворяющих условию $x \in D$, была посвящена работа [4], а немного ранее в [10] (в контексте изучения распределения числа точек случайного линейного подпространства, попавших в заданное множество) был рассмотрен частный случай, когда $D=V^{n} \backslash\left\{0^{n}\right\}$. Исследованию числа решений случайных систем линейных уравнений, принадлежащих заданному множеству $D$, были посвящены работы $[2-4,12]$.

В настоящей работе мы рассматриваем обобщение этой задачи, считая, что правая часть включения (множество $B$ ) зависит от вектора $x$. Таким образом, мы приходим к паре соотношений

$$
x \in D, \quad A x \in B(x),
$$

где множество $B(x)$ является функцией аргумента $x \in D$. Система $x \in D, A x \in B(x)$ эквивалентна одному линейному включению вида

$$
\left(\begin{array}{c}
x \\
A x
\end{array}\right)=\left(\begin{array}{c}
I_{n} \\
A
\end{array}\right) x \in J
$$


размерности $n+T$, где $\left(\begin{array}{l}I \\ A\end{array}\right)-$ матрица размера $(n+T) \times n$ с единичной матрицей $I_{n}$ размера $n$ в качестве верхнего блока,

$$
J=J(D, \bar{B}(D))=\bigcup_{x \in D}\{(x, b): b \in B(x)\} .
$$

Здесь и далее под $\bar{B}(D)$ понимается набор множеств $B(x), x \in D$. По этой причине систему включений (1) мы назвали обобщенным линейным включением. Число решений системы (1) обозначим $\xi(D, A, \bar{B}(D))$.

Мы рассматриваем случай, когда параметры $n$ и $T$ согласованно возрастают: $n, T \rightarrow \infty$. Нас интересуют условия на характер изменения множеств $D=D(n)$ и $B(x)=B(T, x)$ и распределений элементов матрицы $A=A(T, n)$ при $n, T \rightarrow \infty$, обеспечивающие сходимость распределения числа решений системы (1) к сложному пуассоновскому распределению. Аналогичные вопросы для числа решений случайных включений изучались в работах [4-6]. Ниже мы покажем, что новая постановка позволяет получить обобщения некоторых результатов этих работ как следствия предельной теоремы для числа решений системы (1).

Опишем характеристики, в терминах которых будут выписаны условия наших предельных теорем. Прежде всего, предполагается, что элементы случайной матрицы $A=\left\|a_{i, j}\right\|_{T \times n}$ независимы в совокупности и распределены с вероятностями

$$
\mathbf{P}\left\{a_{i, j}=k\right\}=\frac{1+\Delta_{i, j}(k)}{q}, \quad k \in K,
$$

где

$$
\sum_{k \in K} \Delta_{i, j}(k)=0, \quad i=1, \ldots, T, \quad j=1, \ldots, n
$$

Пусть

$$
\Delta=\max _{i, j, k}\left|\Delta_{i, j}(k)\right|<1 .
$$

Следуя [4-6], введем следующее определение. Рассмотрим подмножество $H$ элементов линейного пространства $V^{m}$. Пусть $N\left(a_{1}, a_{2}, a_{3}, d, H\right)$ обозначает число решений системы из $m$ линейных уравнений над полем $K$, записанной в виде уравнения

$$
a_{1} u^{1}+a_{2} u^{2}+a_{3} u^{3}=d
$$

относительно тройки векторов $\left(u^{1}, u^{2}, u^{3}\right) \in H^{3}, a_{1}, a_{2}, a_{3} \in K \backslash\{0\}, d \in V^{m}$. Здесь и далее в аналогичных случаях + обозначает сложение векторов в линейных пространствах над полем $K$. Положим

$$
\begin{aligned}
N(H) & =\max _{a_{1}, a_{2}, a_{3}, d} N\left(a_{1}, a_{2}, a_{3}, d, H\right), \\
\rho(H) & =\frac{N(H)}{|H|^{2}},
\end{aligned}
$$

где $|H|$ обозначает число элементов конечного множества $H$. Очевидно, что

$$
0 \leqslant \rho(H) \leqslant 1
$$


Эта характеристика сначала была использована в [10] (для множества $B$ ) и потом в [4-6] (применительно к множествам $D$ и $B$ ) как одно из существенных условий предельных теорем. Она является своего рода мерой отличия множества $H$ от линейного или аффинного пространства. Для линейных и аффинных подпространств эта мера принимает максимально возможное значение $\rho(H)=1$ (см. [12]). Условие $\rho(H) \rightarrow 0$ можно трактовать как асимптотическую свободу множества $H=H(m)$ от линейных комбинаций своих элементов при $m \rightarrow \infty$. Непосредственно из определения величины $\rho(H)$ вытекают следующие свойства.

(А) При любых $h \in V^{m}$ выполнено равенство

$$
\rho(H+h)=\rho(H) .
$$

(Б) Из соотношения $\rho(H) \rightarrow 0$ при $m \rightarrow \infty$ вытекает соотношение $\rho\left(H \cup H^{\prime}\right) \rightarrow 0$ для любого множества $H^{\prime}$, удовлетворяющего условию $\left|H^{\prime}\right| /|H| \rightarrow 0$ при $T \rightarrow \infty$.

Элементы $(x, b)$ и $\left(x^{\prime}, b^{\prime}\right)$ назовем подобными, если

$$
\left(x^{\prime}, b^{\prime}\right)=(k x, k b)
$$

при некотором элементе $k \in K \backslash\{0\}$.

Разобьем множество $J$ на классы $J_{1}, \ldots, J_{M}$ подобных векторов, где $M$ - общее число таких классов. Положим

$$
l_{u}(J)=\left|\left\{k \in\{1, \ldots, M\}:\left|J_{k}\right|=u\right\}\right|, \quad u=1, \ldots, q-1 .
$$

Пусть $\pi_{1}\left(\lambda_{1}\right), \pi_{2}\left(\lambda_{2}\right), \ldots, \pi_{q-1}\left(\lambda_{q-1}\right)$ - независимые случайные величины, распределенные по закону Пуассона с параметрами $\lambda_{1}, \lambda_{2}, \ldots, \lambda_{q-1}$ соответственно.

Теорема 1. Пусть $K=G F(q)$, распределение элементов матрицы $А$ удовлетворяет условию (4), $0^{n} \notin D$, и при $n, T \rightarrow \infty$ выполнень соотночения $|D| \rightarrow \infty$,

$$
\begin{aligned}
\frac{l_{r}(J)}{q^{T}} \rightarrow \lambda_{r}, \quad 0 \leqslant \lambda_{r}<\infty, \quad r & =1, \ldots, q-1, \quad \exists r: \quad \lambda_{r}>0, \\
\frac{1}{q^{T}} \max _{x \in D}|B(x)|=|D|^{\varepsilon(n)-1}, \quad \varepsilon(n)=o(1), & \\
\rho(J) & \rightarrow 0, \\
T \Delta & \rightarrow 0
\end{aligned}
$$

Тогда распределение случайной величины $\xi(D, A, \bar{B}(D))$ сходится к распределению случайной величинь

$$
\pi\left(\lambda_{1}, \ldots, \lambda_{q-1}\right)=\pi\left(\lambda_{1}\right)+2 \pi\left(\lambda_{2}\right)+\ldots+(q-1) \pi\left(\lambda_{q-1}\right) .
$$

Замечание 1. Условие (9) используется впервые. Условие (10) было введено в [2] при изучении числа специальных решений случайных систем линейных уравнений над полем $G F(2)$. Оно использовалось также в [3-6].

Замечание 2. Из условия (7) и определений (6) следует, что величина $|J|$ имеет при $n, T \rightarrow \infty$ такой же порядок роста, как и $q^{T}$. Поэтому условие (8) в теореме 1 эквивалентно соотношениям

$$
\begin{aligned}
\max _{x \in D}|B(x)| & =C(n) \frac{|J|}{|D|}=\frac{C(n)}{|D|} \sum_{x \in D}|B(x)|, \\
C(n) & =\exp \{o(\ln |D|)\} .
\end{aligned}
$$


Если числа $|B(x)|$ одинаковы при всех $x \in D$, то соотношения (12) выполнены с $C(n)=1$. Значит, в этом случае условие (8) теоремы 1 также выполнено, и его можно опустить.

Рассмотрим теперь систему включений

$$
x \in D, \quad A x+S(x) \in B,
$$

где $S(x)$ - произвольное отображение $V^{n}$ в $V^{T}$, а $B=B(T)-$ заданная последовательность множеств векторов пространств $V^{T}$. Через $\xi(D, A+S, B)$ обозначим число ее решений. Система (13) может быть преобразована в систему $x \in D, A x \in B(x)$, для которой

$$
B(x)=\{x: b+S(x) \in B\}, \quad J=\bigcup_{x \in D}\{(x, b): b+S(x) \in B\} .
$$

Для числа решений системы (13) с помощью теоремы 1 выведем следующее утверждение.

Теорема 2. Пусть $K=G F(q)$, распределение элементов матрищь А удовлетворяет условию (4), $0^{n} \notin D$, и при $n, T \rightarrow \infty$ выполнень соотношения $|D| \rightarrow \infty$, (7), (10),

$$
\rho(D) \rho(B) \rightarrow 0 \text {. }
$$

Тогда распределение случайной величины $\xi(D, A+S, B)$ сходится к распределению случайной величины $\pi\left(\lambda_{1}, \ldots, \lambda_{q-1}\right)$.

Замечание 3. Одно из отличий теоремы 2 от теоремы 1 состоит в том, что в теореме 2 условие $\rho(J) \rightarrow 0$ заменено условием (14), которое легко проверить в тех случаях, когда известна асимптотика хотя бы одного из сомножителей в левой части. Это удобно, например, если одно из множеств $D$ или $B$ является шаром или сферой в метрике Хэмминга (их свойства описаны в $[4,7]$.

Замечание 4. Теорема 2 распространяет на наш случай теорему 3 в [4], в которой отображение $S \equiv 0^{T}$ (с сохранением условий (7), (10) и (14)).

Замечание 5. Если множество $B$ состоит из единственного вектора $b$, то утверждение теоремы 2 относится к числу специальных решений системы уравнений $F(x)=b$, где $F(x)=A x+S(x)$, со случайными линейными частями $A x$. В этом случае условие (14) заменяется условием $\rho(D) \rightarrow 0$.

Замечание 6. Если в множестве $J$ нет подобных векторов, то условие (7) превращается в условие $q^{-T}|J| \rightarrow \lambda \in(0, \infty)$, а предельным в теоремах 1 и 2 будет распределение Пуассона с параметром $\lambda$. Так будет, например, если $K=G F(2)$.

Наконец, рассмотрим систему включений (13) с произвольным случайным отображением $S(x)$ пространства $V^{n}$ в $V^{T}$, которое не зависит от случайной матрицы $A$.

Теорема 3. Пусть $K=G F(q)$, множество $D$ не содержит подобньх векторов, отображение $S(x)$ не зависит от матрицы $A$, распределение элементов матрицы А удовлетворяет условию (4), $0^{n} \notin D$, и при $n, T \rightarrow \infty$ выполнены соотношения $|D| \rightarrow \infty$, (10), (14) $u$

$$
q^{-T}|D||B| \rightarrow \lambda \in(0, \infty) .
$$

Тогда распределение случайной величины $\xi(D, A+S, B)$ сходится к распределению Пуассона с параметром $\lambda$, причем эта сходимость равномерна относительно распределения отображения $S(x)$. 
Замечание 7. Теорема 3 , в отличие от теоремы 1 , относится к нелинейному включению общего вида $F(x) \in B$ со случайным отображением $F: V^{n} \rightarrow V^{T}$ и одинаковым для всех $x \in D$ множеством $B$. В частности, в качестве компонент отображения $F(x)$ могут использоваться полиномы любых степеней. При этом предполагается, что отображение $F(x)$ имеет независимую линейную часть $A x$, в которой распределение матрицы $A$ удовлетворяет условию асимптотической равномерности $T \Delta \rightarrow 0$.

\section{2. Доказательство теоремы 1}

Пусть $I\{E\}$ означает индикатор случайного события $E$. Рассмотрим систему случайных индикаторов $\{I\{A x=b\}:(x, b) \in \bar{J}\}$, где $\bar{J} \subseteq J$. Пусть задан набор $R_{1}, \ldots, R_{S}$ непересекающихся подмножеств множества $\bar{J}$. Пусть

$$
\xi_{u}=\sum_{(x, b) \in R_{u}} I\{A x=b\}, \quad u=1, \ldots, s .
$$

Теорема 4. Пусть $K=G F(q)$, распределение элементов матриць $А$ удовлетворяет условию (4), $0^{n} \notin D$, множество $\bar{J}$ не содержит подобных векторов, и при $n, T \rightarrow \infty$ выполнены условия $|D| \rightarrow \infty$, (8), $\rho(\bar{J}) \rightarrow 0, T \Delta \rightarrow 0$,

$$
\begin{gathered}
q^{-T}|J|=q^{-T} \sum_{x \in D}|B(x)|=O(1), \\
q^{-T}\left|R_{u}\right| \rightarrow \lambda_{u}^{\prime}, \quad 0 \leqslant \lambda_{u}^{\prime}<\infty, \quad u=1, \ldots, s .
\end{gathered}
$$

Тогда случайные величины $\xi_{1}, \ldots, \xi_{\text {s }}$ асимптотически независимы, а их распределения сходятся к распределениям Пуассона с параметрами $\lambda_{1}^{\prime}, \ldots, \lambda_{s}^{\prime}$ соответственно.

Доказательство. Воспользуемся многомерной версией теоремы Б. А. Севастьянова о предельном распределении Пуассона суммы зависимых индикаторов (см. [11]). Для ее применения потребуется ряд определений. Введем множества

$$
\begin{aligned}
D_{k, j} & =\left\{\left(x^{1}, \ldots, x^{k}\right) \in D^{k}: \operatorname{rank}\left\langle x^{1}, \ldots, x^{k}\right\rangle=j\right\}, \\
D_{k} & =\bigcup_{j=1}^{k-1} D_{k, j} .
\end{aligned}
$$

Заметим, что

$$
\left|D_{k, j}\right| \leqslant\left(\begin{array}{c}
k \\
j
\end{array}\right) q^{j(k-j)}|D|^{j} .
$$

Положим

$$
\bar{J}_{k}=\left\{\left(\left(x^{1}, b^{1}\right), \ldots,\left(x^{k}, b^{k}\right)\right) \in \bar{J}^{k}:\left(x^{\alpha}, b^{\alpha}\right) \neq\left(x^{\beta}, b^{\beta}\right) \text { при } \alpha \neq \beta\right\} .
$$

Определим исключительные множества $I_{k} \subset \bar{J}_{k}$ равенством

$$
I_{k}=\left\{\left(\left(x^{1}, b^{1}\right), \ldots,\left(x^{k}, b^{k}\right)\right) \in \bar{J}_{k}:\left(x^{1}, \ldots, x^{k}\right) \in D_{k}\right\} .
$$


Теорема будет доказана, если убедиться, что

$$
\begin{aligned}
& \sum_{(x, b) \in R_{u}} \mathbf{P}\{A x=b\} \rightarrow \lambda_{u}^{\prime}, \quad u=1, \ldots, s, \\
& \max _{(x, b) \in \bar{J}} \mathbf{P}\{A x=b\} \rightarrow 0,
\end{aligned}
$$

и при всех $k=2,3, \ldots$ (используем обозначение $\left.v^{i}=\left(x^{i}, b^{i}\right)\right)$

$$
\begin{array}{r}
\max _{\left(v^{1}, \ldots, v^{k}\right) \in \bar{J}_{k} \backslash I_{k}}\left|\frac{\mathbf{P}\left\{A x^{1}=b^{1}, \ldots, A x^{k}=b^{k}\right\}}{\mathbf{P}\left\{A x^{1}=b^{1}\right\} \cdots \mathbf{P}\left\{A x^{k}=b^{k}\right\}}-1\right| \rightarrow 0, \\
\sum_{\left(v^{1}, \ldots, v^{k}\right) \in I_{k}} \prod_{i=1}^{k} \mathbf{P}\left\{A x^{j}=b^{j}\right\} \rightarrow 0, \\
\sum_{\left(v^{1}, \ldots, v^{k}\right) \in I_{k}} \mathbf{P}\left\{A x^{1}=b^{1}, \ldots, A x^{k}=b^{k}\right\} \rightarrow 0 .
\end{array}
$$

Перед проверкой условий (20)-(24) приведем вспомогательное утверждение.

Лемма 1. Пусть $\left(x^{1}, \ldots, x^{k}\right) \in D_{k, j}, b^{1}, \ldots, b^{k} \in V^{T}$ и выполнено условие (4). Тогда при всех $k=1,2, \ldots$

$$
\mathbf{P}\left\{A x^{1}=b^{1}, \ldots, A x^{k}=b^{k}\right\} \leqslant\left(\frac{1+\Delta}{q}\right)^{j T},
$$

если $1 \leqslant j \leqslant k-1$, а если $j=k$, то

$$
\left(\frac{1-\Delta}{q}\right)^{k T} \leqslant \mathbf{P}\left\{A x^{1}=b^{1}, \ldots, A x^{k}=b^{k}\right\} \leqslant\left(\frac{1+\Delta}{q}\right)^{k T}
$$

Эта лемма повторяет лемму 3 из [3].

Теперь соотношения (20)-(22) следуют из леммы 1 и условий $T \Delta \rightarrow 0$ и (17). Подробности можно найти в [4-6].

Проверим соотношение (23) при $k \geqslant 2$. Для этого используем неравенство (25) (где $k=1)$ и получаем

$$
\sum_{\left(v^{1}, \ldots, v^{k}\right) \in I_{k}} \prod_{s=1}^{k} \mathbf{P}\left\{A x^{s}=b^{s}\right\} \leqslant \sum_{j=1}^{k-1}\left(\frac{1+\Delta}{q}\right)^{k T} \sum_{\left(x^{1}, \ldots, x^{k}\right) \in D_{k, j}} \prod_{s=1}^{k}\left|B\left(x_{s}\right)\right| .
$$

Теперь, используя вытекающее из условий (7) и (8) (см. замечание 2) неравенство

$$
\prod_{s=1}^{k}\left|B\left(x_{s}\right)\right| \leqslant\left(C(n) \frac{1}{|D|} \sum_{x \in D}|B(x)|\right)^{k}=\left(\frac{C(n)|J|}{|D|}\right)^{k}
$$


и (19), получаем

$$
\begin{aligned}
\sum_{\left(v^{1}, \ldots, v^{k}\right) \in I_{k}} \prod_{s=1}^{k} \mathbf{P}\left\{A x^{s}=b^{s}\right\} \leqslant & \left(\frac{C(n)|J|}{|D|}\right)^{k}\left(\frac{1+\Delta}{q}\right)^{k T} \sum_{j=1}^{k-1}\left|D_{k, j}\right| \\
\leqslant & \left(\frac{|J|}{q^{T}}\right)^{k}(1+\Delta)^{k T} \\
& \times \frac{(C(n))^{k}}{|D|} \frac{1}{|D|^{k-1}} \sum_{j=1}^{k-1}\left(\begin{array}{l}
k \\
j
\end{array}\right) q^{j(k-j)}|D|^{j} .
\end{aligned}
$$

Из условий (16), (8) (см. замечание 2), $T \Delta \rightarrow 0$ следует, что выражение в правой части цепочки соотношений (28) стремится к нулю при всех $k \geqslant 2$. Соотношение (23) доказано.

Наконец, проверим соотношение (24). Введем множества

$\bar{D}_{k, j}=D_{k, j} \cap\left\{\left(x^{1}, \ldots, x^{k}\right) \in D^{k}: x^{\alpha} \neq c x^{\beta}\right.$ для всех $c \in K \backslash\{0\}$, где $\left.\alpha \neq \beta\right\}$.

Заметим, что

$$
\bar{D}_{k, 1}=\varnothing, \quad k=2,3, \ldots
$$

Положим

$$
\bar{D}_{k}=\bigcup_{j=2}^{k-1} \bar{D}_{k, j}
$$

Так как множество $\bar{J}$ не содержит подобных векторов, то

$$
\sum_{\left(v^{1}, \ldots, v^{k}\right) \in I_{k}} \mathbf{P}\left\{A x^{1}=b^{1}, \ldots, A x^{k}=b^{k}\right\}=\sum_{\substack{\left(v^{1}, \ldots, v^{k}\right) \in \bar{J}_{k} \\\left(x^{1}, \ldots, x^{k}\right) \in \bar{D}_{k}}} \mathbf{P}\left\{A x^{1}=b^{1}, \ldots, A x^{k}=b^{k}\right\} .
$$

Отсюда следует, что

$$
\sum_{\left(v^{1}, v^{2}\right) \in I_{2}} \mathbf{P}\left\{A x^{1}=b^{1}, A x^{2}=b^{2}\right\}=0 .
$$

Поэтому далее мы считаем, что $k \geqslant 3$.

Сопоставим набору $x^{1}, \ldots, x^{k}$ векторов пространства $V^{n}$ систему $L_{x}\left(x^{1}, \ldots, x^{k}\right)$ однородных линейных уравнений вида

$$
\alpha_{1} x^{1}+\ldots+\alpha_{k} x^{k}=0
$$

возможно, пустую, составленную из всех линейных соотношений, которым удовлетворяет набор $x^{1}, \ldots, x^{k}$. Системе $L_{x}\left(x^{1}, \ldots, x^{k}\right)$ сопоставим идентичную по составу систему $L_{b}\left(x^{1}, \ldots, x^{k}\right)$ из линейных уравнений относительно $b^{1}, \ldots, b^{k} \in V^{T}$. Обозначим через $B\left(x^{1}, \ldots, x^{k}\right)$ множество решений $\left(b^{1}, \ldots, b^{k}\right)$ системы $L_{b}\left(x^{1}, \ldots, x^{k}\right)$, удовлетворяющих условию $b^{1} \in B\left(x^{1}\right), \ldots, b^{k} \in B\left(x^{k}\right)$.

Лемма 2. Пусть $b^{1} \in B\left(x^{1}\right), \ldots, b^{k} \in B\left(x^{k}\right),\left(b^{1}, \ldots, b^{k}\right) \notin B\left(x^{1}, \ldots, x^{k}\right),\left(x^{1}, \ldots, x^{k}\right) \in$ $\bar{D}_{k}$. Тогда

$$
\mathbf{P}\left\{A x^{1}=b^{1}, \ldots, A x^{k}=b^{k}\right\}=0 .
$$


Доказательство. Предположим противное, а именно, что для некоторых наборов векторов $\left(x^{1}, \ldots, x^{k}\right) \in \bar{D}_{k}$ и $\left(b^{1}, \ldots, b^{k}\right) \notin B\left(x^{1}, \ldots, x^{k}\right), b^{1} \in B\left(x^{1}\right), \ldots, b^{k} \in B\left(x^{k}\right)$, выполнено неравенство

$$
\mathbf{P}\left\{A x^{1}=b^{1}, \ldots, A x^{k}=b^{k}\right\}>0 \text {. }
$$

Тогда найдется такой набор элементов поля $\left(\alpha_{1}, \ldots, \alpha_{k}\right) \neq(0, \ldots, 0)$, что

$$
\begin{aligned}
& \alpha_{1} x^{1}+\ldots+\alpha_{k} x^{k}=0, \\
& \alpha_{1} b^{1}+\ldots+\alpha_{k} b^{k} \neq 0,
\end{aligned}
$$

и, кроме этого, для некоторой реализации $A^{\prime}$ случайной матрицы $A$ будет выполнен набор равенств

$$
A^{\prime} x^{1}=b^{1}, \ldots, A^{\prime} x^{k}=b^{k} .
$$

Очевидно, что условия (32) и (33) не могут выполняться одновременно. Следовательно,

$$
\mathbf{P}\left\{A x^{1}=b^{1}, \ldots, A x^{k}=b^{k}\right\}=0 .
$$

Лемма 2 доказана.

Из леммы 2 следует, что

$$
\begin{aligned}
& \sum_{\substack{\left(v^{1}, \ldots, v^{k}\right) \in \bar{J}_{k} \\
\left(x^{1}, \ldots, x^{k}\right) \in \bar{D}_{k}}} \mathbf{P}\left\{A x^{1}=b^{1}, \ldots, A x^{k}=b^{k}\right\} \\
& \quad=\sum_{j=2}^{k-1} \sum_{\left(x^{1}, \ldots, x^{k}\right) \in \bar{D}_{k, j}} \sum_{\left(b^{1}, \ldots, b^{k}\right) \in B\left(x^{1}, \ldots, x^{k}\right)} \mathbf{P}\left\{A x^{1}=b^{1}, \ldots, A x^{k}=b^{k}\right\} .
\end{aligned}
$$

Лемма 3. Пусть $2 \leqslant j \leqslant k-1$. Тогда

$$
\left|\left\{\left(x^{1}, \ldots, x^{k}\right) \in \bar{D}_{k, j},\left(b^{1}, \ldots, b^{k}\right) \in B\left(x^{1}, \ldots, x^{k}\right)\right\}\right| \leqslant \rho(\bar{J})|\bar{J}|^{j} .
$$

Доказательство. Так как

$$
\operatorname{rank}\left\langle x^{1}, \ldots, x^{k}\right\rangle \leqslant k-1
$$

то системы $L_{x}\left(x^{1}, \ldots, x^{k}\right)$ и $L_{b}\left(x^{1}, \ldots, x^{k}\right)$ не пусты. Перенумеруем векторы $x^{1}, \ldots, x^{k}$ так, чтобы первые $j$ из них составляли базис. Преобразованные системы $L_{x}\left(x^{1}, \ldots, x^{k}\right)$ и $L_{b}\left(x^{1}, \ldots, x^{k}\right)$ будут содержать, соответственно, уравнения

$$
\begin{aligned}
& x^{j+1}=l_{j+1}\left(x^{1}, \ldots, x^{j}\right), \\
& b^{j+1}=l_{j+1}\left(b^{1}, \ldots, b^{j}\right),
\end{aligned}
$$

где $l_{j+1}-$ однородная линейная функция своих аргументов. При этом значения векторов $x^{j+1}, \ldots, x^{k}$ и $b^{j+1}, \ldots, b^{k}$ определяются однозначно векторами $x^{1}, \ldots, x^{j}$ и $b^{1}, \ldots, b^{j}$ соответственно.

Так как $\left(x^{1}, \ldots, x^{k}\right) \in \bar{D}_{k, j}$, то в системах $L_{x}\left(x^{1}, \ldots, x^{k}\right)$ и $L_{b}\left(x^{1}, \ldots, x^{k}\right)$ нет одночленных и двучленных уравнений. Значит, каждое из уравнений (35) содержит по крайней 
мере три существенные переменные. Среди них заведомо есть переменные $x^{j+1}$ и $b^{j+1}$. Не нарушая общности рассуждений, можно считать, что проведенная перенумерация векторов была такой, что существенными в этих уравнениях являются также переменные $x^{1}$, $x^{2}$ и $b^{1}, b^{2}$ соответственно. Значит, эти уравнения можно переписать в виде

$$
\begin{aligned}
& x^{j+1}=a_{1} x^{1}+a_{2} x^{2}+l_{j+1}^{\prime}\left(x^{3}, \ldots, x^{j}\right), \\
& b^{j+1}=a_{1} b^{1}+a_{2} b^{2}+l_{j+1}^{\prime}\left(b^{3}, \ldots, b^{j}\right),
\end{aligned}
$$

с некоторыми ненулевыми коэффициентами $a_{1}$ и $a_{2}$.

Зафиксируем значения переменных $x^{3}, \ldots, x^{j}$ и $b^{3} \in B\left(x^{3}\right), \ldots, b^{j} \in B\left(x^{j}\right)$. Число решений системы уравнений (36) при такой фиксации не превосходит

$$
N(\bar{J})=\max _{a_{1}, a_{2}, a_{3}, d} N\left(a_{1}, a_{2}, a_{3}, d, \bar{J}\right),
$$

где $N\left(a_{1}, a_{2}, a_{3}, d=\left(d^{\prime}, d^{\prime \prime}\right), \bar{J}\right)$ обозначает число решений системы из $n+T$ линейных уравнений над полем $K$, записанной в виде пары уравнений

$$
\begin{aligned}
& x^{j+1}=a_{1} x^{1}+a_{2} x^{2}+d^{\prime}, \\
& b^{j+1}=a_{1} b^{1}+a_{2} b^{2}+d^{\prime \prime} .
\end{aligned}
$$

Согласно определениям, приведенным во Введении,

$$
N(\bar{J})=\rho(\bar{J})|\bar{J}|^{2} .
$$

Заметим еще, что система (36) допускает не более $|\bar{J}|^{j-2}$ вариантов задания переменных $x^{3}, \ldots, x^{j}$ и $b^{3} \in B\left(x^{3}\right), \ldots, b^{j} \in B\left(x^{j}\right)$. Поэтому число ее решений оценивается сверху величиной $\rho(\bar{J})|\bar{J}|^{j}$. Лемма 3 доказана.

Применим лемму 3 и оценку (26) к выражению в правой части (34), а результат подставим в (30). Получим, учитывая условия теоремы 4, что

$$
\begin{aligned}
& \sum_{\left(v^{1}, \ldots, v^{k}\right) \in I_{k}} \mathbf{P}\left\{A x^{1}=b^{1}, \ldots, A x^{k}=b^{k}\right\} \\
& \leqslant \sum_{j=2}^{k-1} \sum_{\left(x^{1}, \ldots, x^{k}\right) \in \bar{D}_{k, j}} \sum_{\left(b^{1}, \ldots, b^{k}\right) \in B\left(x^{1}, \ldots, x^{k}\right)}\left(\frac{1+\Delta}{q}\right)^{j T} \\
& =\sum_{j=2}^{k-1}\left(\frac{1+\Delta}{q}\right)^{j T}\left|\left\{\left(x^{1}, \ldots, x^{k}\right) \in \bar{D}_{k},\left(b^{1}, \ldots, b^{k}\right) \in B\left(x^{1}, \ldots, x^{k}\right)\right\}\right| \\
& \leqslant \rho(\bar{J}) \sum_{j=2}^{k-1}\left(\frac{1+\Delta}{q}\right)^{j T}|J|^{j}=O\left(\rho(\bar{J}) \sum_{j=2}^{k-1}\left(\frac{|J|}{q^{T}}\right)^{j}\right)=O(\rho(\bar{J})) \rightarrow 0 .
\end{aligned}
$$

Значит, условие (24) выполнено. Итак, мы проверили все условия многомерной версии теоремы Б. А. Севастьянова, и можем воспользоваться ею. Теорема 4 доказана.

Доказательство теоремь 1. Напомним, что согласно (6)

$$
l_{u}(J)=\left|\left\{k \in\{1, \ldots, M\}:\left|J_{k}\right|=u\right\}\right|, \quad u=1, \ldots, q-1,
$$


где $J_{1}, \ldots, J_{M}$ - разбиение множества $J$ на классы подобных векторов и $M-$ общее число таких классов. Выберем из каждого множества $J_{k}$ по одному произвольному элементу $\omega_{k} \in J_{k}$ и положим

$$
\bar{J}=\left\{\omega_{1}, \omega_{2}, \ldots, \omega_{M}\right\} .
$$

Построим разбиение этого множества на непересекающиеся множества $R_{1}, \ldots, R_{q-1}$ по правилу

$$
R_{u}=\left\{\omega_{k}: k \in\{1, \ldots, M\},\left|J_{k}\right|=u\right\}, \quad u=1, \ldots, q-1,
$$

и введем случайные величины

$$
\xi_{u}=\sum_{(x, b) \in R_{u}} I\{A x=b\}, \quad u=1, \ldots, q-1,
$$

где множества $R_{1}, \ldots, R_{q-1}$ определены правилом (38). Тогда

$$
\xi(D, A, \bar{B})=\xi_{1}+2 \xi_{2}+\ldots+(q-1) \xi_{q-1} .
$$

Согласно определению,

$$
\rho(\bar{J})=\frac{N(\bar{J})}{|\bar{J}|^{2}}
$$

где

$$
N(\bar{J}) \leqslant N(J), \quad|\bar{J}| \geqslant \frac{|J|}{q-1} .
$$

Поэтому

$$
\rho(\bar{J}) \leqslant \frac{\rho(J)}{(q-1)^{2}},
$$

и условие (9) теоремы 1 влечет условие $\rho(\bar{J}) \rightarrow 0$ теоремы 4. Значит, для случайных величин (39) выполнены все условия теоремы 4, причем

$$
\lambda_{u}^{\prime}=\lambda_{u}, \quad u=1, \ldots, q-1 .
$$

Для завершения доказательства теоремы 1 остается воспользоваться равенством (40).

\section{3. Доказательство теоремы 2}

Как уже отмечалось во Введении, систему (13) можно записать как

$$
x \in D, \quad A x \in B(x)
$$

с множествами

$$
\begin{aligned}
B(x) & =\{x: b+S(x) \in B\}, \\
J=J(D, \bar{B}(D)) & =\bigcup_{x \in D}\{(x, b): b+S(x) \in B\} .
\end{aligned}
$$

Покажем, что для этой системы выполнены условия теоремы 1 . Заметим, что $|B(x)|$ одинаковы и равны $|B|$. Поэтому выполнено условие (8) (см. замечание 2). Остальные условия теоремы 1 , кроме соотношения (9), присутствуют в условиях доказываемой теоремы. Итак, нам осталось показать, что из (14) следует (9). 
Лемма 4. Для множества $J$ вида (42) выполнено неравенство

$$
\rho(J) \leqslant \rho(D) \rho(B) .
$$

Нам понадобится следующее определение. Рассмотрим подмножества $B_{1}, B_{2}, B_{3}$ элементов пространства $V^{T}$. Пусть $N\left(a_{1}, a_{2}, a_{3}, d, B_{1}, B_{2}, B_{3}\right)$ обозначает число решений системы из $T$ линейных уравнений над полем $K$, записанной в виде уравнения

$$
a_{1} u^{1}+a_{2} u^{2}+a_{3} u^{3}=d,
$$

относительно тройки векторов $\left(u^{1}, u^{2}, u^{3}\right) \in B_{1} \times B_{2} \times B_{3}, a_{1}, a_{2}, a_{3} \in K \backslash\{0\}, d \in V^{T}$. Положим

$$
\begin{aligned}
& N\left(B_{1}, B_{2}, B_{3}\right)=\max _{a_{1}, a_{2}, a_{3}, d} N\left(a_{1}, a_{2}, a_{3}, d, B_{1}, B_{2}, B_{3}\right), \\
& \rho\left(B_{1}, B_{2}, B_{3}\right)=\frac{N\left(B_{1}, B_{2}, B_{3}\right)}{\left|B_{1}\right|\left|B_{2}\right|},
\end{aligned}
$$

Из этого определения и определения величины $\rho(B)$, приведенного во Введении, вытекают следующие свойства:

$$
\begin{aligned}
\rho(B, B, B) & =\rho(B), \\
\rho\left(B_{1}+b^{1}, B_{2}+b^{2}, B_{3}+b^{3}\right) & =\rho\left(B_{1}, B_{2}, B_{3}\right) \quad \text { для всех } b^{1}, b^{2}, b^{3} \in V^{T} ;
\end{aligned}
$$

в частности,

$$
\rho\left(B+b^{1}, B+b^{2}, B+b^{3}\right)=\rho(B) .
$$

Доказательство леммы 4. Рассмотрим систему уравнений

$$
a_{1} u^{1}+a_{2} u^{2}+a_{3} u^{3}=d,
$$

относительно тройки векторов $\left(u^{1}, u^{2}, u^{3}\right) \in J^{3}, a_{1}, a_{2}, a_{3} \in K \backslash\{0\}, d \in V^{n+T}$. Здесь $u^{j}=\left(x^{j}, b^{j}\right), j=1,2,3$. Согласно (42) систему (46) можно рассматривать как объединение двух систем: системы

$$
a_{1} x^{1}+a_{2} x^{2}+a_{3} x^{3}=d^{X}
$$

относительно $\left(x^{1}, x^{2}, x^{3}\right) \in D^{3}$, и системы

$$
a_{1} b^{1}+a_{2} b^{2}+a_{3} b^{3}=d^{B}
$$

относительно $b^{1} \in B\left(x^{1}\right), b^{2} \in B\left(x^{2}\right), b^{3} \in B\left(x^{3}\right)$. Здесь

$$
\left(d^{X}, d^{B}\right)=d .
$$

При фиксированных значениях $\left(x^{1}, x^{2}, x^{3}\right) \in D^{3}$ система (48) имеет не более $\rho\left(B\left(x^{1}\right), B\left(x^{2}\right), B\left(x^{3}\right)\right)\left|B\left(x^{1}\right)\right|\left|B\left(x^{2}\right)\right|$ решений (см. формулу (44)). В силу (42), при всех $j=1,2,3$ имеют место равенства

$$
\left|B\left(x^{j}\right)\right|=\left|B+R\left(x^{j}\right)\right|=|B| .
$$


Согласно (45), выполняется равенство

$$
\rho\left(B\left(x^{1}\right), B\left(x^{2}\right), B\left(x^{3}\right)\right)=\rho(B) .
$$

Поэтому при любой фиксации $\left(x^{1}, x^{2}, x^{3}\right) \in D^{3}$ число решений системы (48) не превосходит $\rho(B)|B|^{2}$.

Значит, число решений системы (46) не превосходит произведения числа решений системы (47), которое оценивается сверху величиной $\rho(D)|D|^{2}$, и выражения $\rho(B)|B|^{2}$. Значит, согласно (5),

$$
\rho(J) \leqslant \frac{\rho(D) \rho(B)|D|^{2}|B|^{2}}{|J|^{2}} .
$$

Так как все $|B(x)|$ одинаковы и равны $|B|$, то

$$
|J|=|D||B|
$$

Поэтому из (49) следует (43). Лемма 4 доказана.

Таким образом, все условия теоремы 1 выполнены, и мы можем применить ее к системе (41). Теорема 2 доказана.

\section{4. Доказательство теоремы 3}

Пусть классическая (одномерная) теорема Б. А. Севастьянова (см. [1]) применяется к случайной величине $W_{m}(\theta)$, являющейся суммой случайных индикаторов, совместное распределение которых зависит от некоторого параметра $\theta \in \Theta$, где $\Theta-$ множество произвольной природы. Если все условия теоремы Б. А. Севастьянова при $m \rightarrow \infty$ выполнены равномерно по $\theta \in \Theta$, а именно, для математического ожидания вместо условия $\mathbf{E} W(\theta) \rightarrow \lambda$ имеем соотношение

$$
\sup _{\theta \in \Theta}|\mathbf{E} W(\theta)-\lambda| \rightarrow 0,
$$

а в остальных условиях производится аналогичная замена, то, как вытекает из доказательства этой теоремы (опять см. [1]), факториальные моменты случайной величины $W_{m}(\theta)$ стремятся к своим пределам также равномерно по $\theta \in \Theta$ :

$$
\sup _{\theta \in \Theta}\left|\mathbf{E} W(\theta)_{r}-\lambda^{r}\right| \rightarrow 0, \quad r=2,3, \ldots
$$

Так как вероятности любой неотрицательной целочисленной случайной величины с любой заранее заданной точностью выражаются через ограниченное число (оно зависит только от точности) факториальных моментов, то получаем при $m \rightarrow \infty$ равномерную сходимость к закону Пуассона в том смысле, что

$$
\sup _{\theta \in \Theta}\left|\mathbf{P}\{W(\theta)=r\}-\frac{\lambda^{r}}{r !} e^{-\lambda}\right| \rightarrow 0, \quad r=0,1, \ldots
$$

Приступим к доказательству теоремы 3. Сначала будем считать, что отображение $S$ является детерминированным. Перепишем систему (13) как

$$
x \in D, \quad A x \in B(x),
$$


где

$$
B(x)=\left\{b \in V^{T}: b+S(x) \in B\right\},
$$

и обратимся к условиям и доказательству теоремы 4, применяя ее к числу решений системы (50). Множество $J$ не содержит подобных векторов (так как их нет в $D$ ). Положим $\bar{J}=J$. Все множества $B(x)$ у нас имеют одинаковую мощность $|B|$, поэтому

$$
|J|=|D||B|
$$

и выполнено условие (8) (см. замечание 2). Возьмем $s=1$. Тогда $R_{1}=J$, а условия (16) и (17) заменятся на (15). Осталось заметить, что, согласно лемме 4, из (14) следует условие $\rho(J) \rightarrow 0$. В итоге получаем, что по предположениям теоремы 3 все условия теоремы 4 выполнены, причем, и это важно, в них не участвует отображение $S$.

Возвращаясь теперь к доказательству теоремы 4, нетрудно убедиться, что построение исключительных множеств ведется одинаково при всех $S$, а все полученные по ходу доказательства оценки, которые затем используются для проверки условий (20)-(24), равномерны по $S$ в указанном выше смысле. Значит, условия теоремы Б. А. Севастьянова выполнены равномерно (в том же смысле) по $S$. Поэтому факториальные моменты и распределение случайной величины $\xi(D, A+S, B)$ при $n, T \rightarrow \infty$ и любом изменении $S$ сходятся к факториальным моментам и распределению закона Пуассона с параметром $\lambda$ равномерно по $S$.

Итак, мы доказали, что

$$
\sup _{S}\left|\mathbf{P}\{\xi(D, A+S, B)=r\}-\frac{\lambda^{r}}{r !} e^{-\lambda}\right| \rightarrow 0 .
$$

Пусть теперь отображение $S$ выбрано случайно и независимо от $A$. Из (51) и формулы полной вероятности следует, что и в этом случае при $n, T \rightarrow \infty$ распределение величины $\xi(D, A+S, B)$ сходится к распределению Пуассона с параметром $\lambda$. Эта сходимость равномерна относительно закона распределения случайного отображения $S$. Теорема 3 доказана.

Замечание 8. Естественным является вопрос о тем, почему в теореме 3 рассмотрен случай сходимости к распределению Пуассона, а не сложному пуассоновскому распределению, как в теоремах 1 и 2. На первый взгляд, использованные рассуждения позволяют это сделать. Проблема здесь заключается в трудности выбора содержательного аналога условия (7) в тех случаях, когда предельное распределение числа решений системы (13) при детерминированном $S(x)$ существенно зависит от $S(x)$. Поэтому мы ограничились случаем, когда для всех $S(x)$ предельное распределение случайной величины $\xi(D, A+S, B)$ одинаково. Это легко достигается с помощью условия об отсутствии подобных векторов в $D$, а оно, в свою очередь, приводит к распределению Пуассона в пределе.

\section{Список литературы}

1. Колчин В. Ф., Севастьянов Б. А., Чистяков В. П., Случайные размещения. Наука, Москва, 1976.

2. Копытцев В. А., О числе решений систем линейных булевых уравнений в множестве векторов, обладающих заданным числом единиц. Дискретная математика (2002) 14, №4, 87-109.

3. Копытцев В. А., О числе решений системы случайных линейных уравнений. Дискретная математика (2006) 18, №1, 40-62. 
4. Копытцев В. А., Михайлов В. Г., Теоремы пуассоновского типа для числа специальных решений случайного линейного включения. Дискретная математика (2010) 22, №2, 3-21.

5. Копытцев В. А., Михайлов В. Г., Теоремы пуассоновского типа для числа решений случайных включений. Математические вопросы криптографии (2010) 1, №4, 63-84.

6. Копытцев В. А., Михайлов В. Г., О распределении чисел решений случайных включений. Математические вопросы криптографии (2011) 2, №2, 55-80.

7. Копытцев В. А., Михайлов В. Г., Об одном асимптотическом свойстве сфер в дискретных пространствах большой размерности. Обозрение прикладной и промышленной математики (2011) 18, №5, 786.

8. Михайлов В. Г., Предельная теорема Пуассона для числа ненулевых решений одной системы случайных уравнений над полем $G F(2)$. Теория вероятностей и ее применения (1998) 43, №3, 598-606.

9. Михайлов В. Г., Предельная теорема Пуассона для числа неколлинеарных решений системы случайных уравнений специального вида. Дискретная математика (2001) 13, №3, 82-90.

10. Михайлов В. Г., Предельные теоремы для числа точек случайного линейного подпространства, попавших в заданное множество. Дискретная математика (2003) 15, №2, 128-137.

11. Михайлов В. Г., О предельной теореме Б. А. Севастьянова для сумм зависимых случайных индикаторов. Обозрение прикладной и промышленной математики (2003) 10, №3, 571-578.

12. Михайлов В. Г., Предельные теоремы для числа решений системы случайных линейных уравнений, попавших в заданное множество. Дискретная математика (2007) 19, №1, 17-26.

Статья поступила 27.03.2012. 